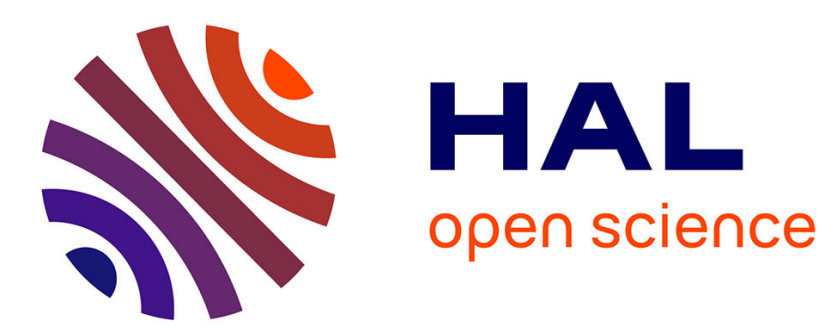

\title{
Classifying DME vs Normal SD-OCT volumes: A review
} Joan Massich, Mojdeh Rastgoo, Guillaume Lemaître, Carol y Cheung, Tien y Wong, Désiré Sidibé, Fabrice Mériaudeau

\section{To cite this version:}

Joan Massich, Mojdeh Rastgoo, Guillaume Lemaître, Carol y Cheung, Tien y Wong, et al.. Classifying DME vs Normal SD-OCT volumes: A review. 23rd International Conference on Pattern Recognition, Dec 2016, Cancun, Mexico. hal-01376469

\section{HAL Id: hal-01376469 \\ https://u-bourgogne.hal.science/hal-01376469}

Submitted on 4 Oct 2016

HAL is a multi-disciplinary open access archive for the deposit and dissemination of scientific research documents, whether they are published or not. The documents may come from teaching and research institutions in France or abroad, or from public or private research centers.
L'archive ouverte pluridisciplinaire HAL, est destinée au dépôt et à la diffusion de documents scientifiques de niveau recherche, publiés ou non, émanant des établissements d'enseignement et de recherche français ou étrangers, des laboratoires publics ou privés. 


\title{
Classifying DME vs Normal SD-OCT volumes: A review
}

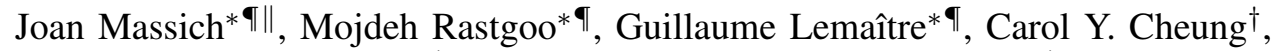 \\ Tien Y. Wong ${ }^{\dagger}$, Désiré Sidibé*, Fabrice Mériaudeau* ${ }^{*}$ \\ *LE2I UMR6306, CNRS, Arts et Métiers, Univ. Bourgogne Franche-Comté, \\ 12 rue de la Fonderie, 71200 Le Creusot, France \\ ${ }^{\dagger}$ Singapore Eye Research Institute, Singapore National Eye Center, Singapore \\ ${ }_{\ddagger}$ Centre for Intelligent Signal and Imaging Research (CISIR), Electrical \& Electronic Engineering Department, \\ Universiti Teknologi Petronas, 32610 Seri Iskandar, Perak, Malaysia \\ IIndicates equal contribution. Ordering determined by Rock-Paper-Scissors-Lizard-Spock.

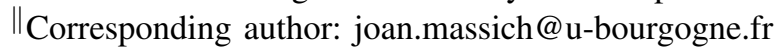

\begin{abstract}
This article reviews the current state of automatic classification methodologies to identify Diabetic Macular Edema (DME) versus normal subjects based on Spectral Domain OCT (SD-OCT) data. Addressing this classification problem has valuable interest since early detection and treatment of DME play a major role to prevent eye adverse effects such as blindness.

The main contribution of this article is to cover the lack of a public dataset and benchmark suited for classifying DME and normal SD-OCT volumes, providing our own implementation of the most relevant methodologies in the literature. Subsequently, 6 different methods were implemented and evaluated using this common benchmark and dataset to produce reliable comparison.

Index Terms-Diabetic Macular Edema (DME), Spectral Domain OCT (SD-OCT), Machine Learning (ML), benchmark,
\end{abstract}

\section{INTRODUCTION}

Diabetic Retinopathy (DR), and more particularly Diabetic Macular Edema (DME), are leading causes of irreversible vision loss and the most common eye diseases in individuals with diabetes. Taking into account that the number of individuals affected by diabetes diseases are expected to grow exponentially in the next decade [1], developing methodologies for early detection and treatment of DR and DME has become a priority to prevent adverse effects.

The main focus of this work is to describe the actual state of DME detection in Optical Coherence Tomography (OCT) images. DME presents an increase in retinal thickness within 1 disk diameter of the fovea center with or without hard exudates and sometimes associated with cysts [2]. Spectral Domain OCT (SD-OCT) is an emerging eye imaging modality providing cross-sectional retinal morphology information [3], which cannot be estimated from more established eye imaging modalities such as fundus imaging.

The initial efforts of the ophtalmic community in developing technologies for SD-OCT have been placed in segmenting the retinal layers, which is a necessary step for retinal thickness measurements [4, 5]. However, latter efforts address the specific problem of DME automatic detection in OCT volumes. These efforts reveal the needs to address: (i) enhancing the quality of OCT volumes, (ii) finding pathology signs, and (iii) appropriate classification strategies.
Advances in any of those regards is of great interest since (i) manual evaluation of SD-OCT volumetric scans is expensive and time consuming [6]; (ii) SD-OCT acquisition has some shortcomings due to eye movements during the scanning [7], reflectivity nature of the retina [8], high level of noise and inconsistent quality of the images; (iii) due to the coexistence of multiple pathologies [7] as well as large intra-pathology variability, consistently identifying pathologyspecific biomarkers remains challenging [6].

The rest of this article is structured as follows: Section $\Pi$ offers a general idea of the literature state-of-the-art in SDOCT volume classification. Section III reviews some publicly available datasets and states the need for another one that suits the classification task here described. Section IV proposes an experimental benchmark to compare different methodologies presented in Sect. III. Section V reports and discusses the obtained results, while Sect.VI wraps up our thoughts regarding this work and its possible direction.

\section{BACKGROUND}

This section reviews works straightly addressing the problem of classifying OCT volumes as normal or abnormal, regardless of the targeted pathology. The methods are categorized in terms of their learning strategy, namely supervised or semi-supervised learning.

\section{A. Supervised methods}

Supervised learning is based on a fully annotated and labeled training set. In this approach, the labeled training data are used to train the classifier function later used for prediction. Figure 11 illustrates a prevalent framework for supervised learning. Each SD-OCT volume undergoes: (i) preprocessing to reduce noise and other acquisition deficiencies which alter the images; (ii) feature detection to quantify visual cues like appearance, texture, shape, etc.; (iii) mapping in which a sample is either considered as whole (i.e., global) or partitioned into a set of sub-elements (i.e., local dense/sparse patches, pyramid, etc.); (iv) feature representation to associate a descriptor (e.g., concatenation, statistics, histogram, 


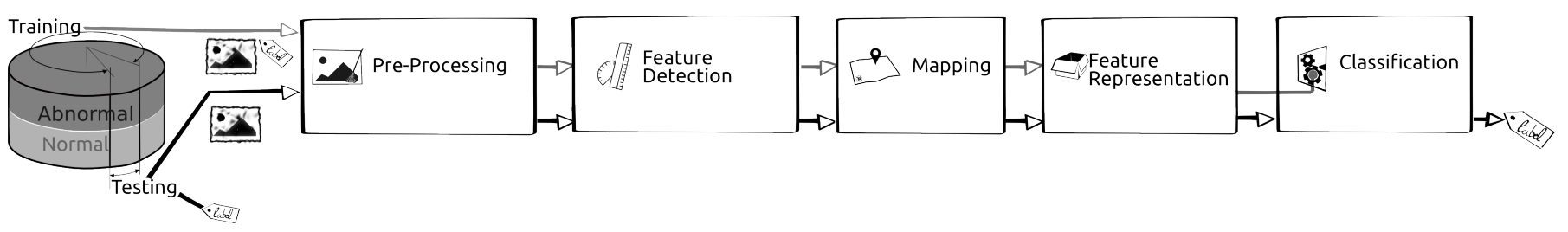

Fig. 1. Common framework

Principal Component Analysis (PCA), Bag-of-Words (BoW), etc.) for each element from the mapping-stage. This descriptor packages the visual cues related to the sample; (v) classification to determine the associated class of each sample.

Venhuizen et al. propose a classification method to distinguish between Age-related Macular Degeneration (AMD) and normal SD-OCT volumes using BoW models [6]. A set of keypoints are detected and selected at each individual B-scan, by keeping the salient points included in the top $3 \%$ of the vertical gradient values. Around each of these keypoints, a $9 \mathrm{px} \times 9 \mathrm{px}$ texton is extracted, generating a feature vector of 81 dimensions, later reduce to 9 using PCA. All extracted feature vectors are used to create a codebook using $k$-means clustering. Then, each OCT volume descriptor is represented as a histogram that captures the codebook occurrences and are classified by a Random Forest (RF) composed of 100 trees. The method is tested using a publicly available dataset of 384 OCT volumes [9], achieving an Area Under the Curve (AUC) of 0.984 .

Srinivasan et al. propose a classification method to distinguish DME, AMD, and normal SD-OCT volumes [10]. Each OCT slice is pre-processed using Block Matching 3D filtering (BM3D) to reduce the speckle noise and is flattened to reduce the inter-patient retinal curvature variations. A multiresolution pyramid is generated for each pre-processed slice and a Histogram of Oriented Gradients (HOG) feature is computed for each layer. These features are classified using a linear Support Vector Machines (SVM). Note that each individual B-scan is classified into one of the three categories, namely DME, AMD, and normal, and a volume is label to a given class by taking the majority vote of all B-scans. This method is also tested using a publicly available dataset, composed of 45 patients equally subdivided into the three targeted classes. Correct classification rates of $100 \%, 100 \%$ and $86.67 \%$ are obtained for normal, DME, and AMD patients, respectively.

Extending the previous work, Alsaih et al. aggregate Local Binary Patterns (LBP) to HOG in order to add texture information and reduce the number of dimension using PCA [11]

Lemaitre et al. propose a method based on LBP features to describe the texture of OCT images and dictionary learning using the BoW models [12]. In this method, the OCT images are first pre-processed using Non-Local Means (NLM) filtering, to reduce the speckle noise. Then, the volumes are mapped into a discrete set of structures: (i) local corresponding to patches, or (ii) global corresponding to volume slices or the whole volume. According to the chosen mapping, LBP or LBP from Three Orthogonal Planes (LBP-TOP) texture features are extracted and represent each volume through histogram, PCA, or BoW representation. The final feature descriptors are classified using RF classifier. This methodology is tested against Venhuizen et al. [6] using public and nonpublic datasets showing an improvement within the results by achieving a Sensitivity (SE) of $87.5 \%$ and a Specificity (SP) of $75 \%$.

Liu et al. propose a methodology aiming at classifying Bscan rather than volume. The classification goal is to distinguish between macular pathology and normal OCT B-scan images using LBP and gradient information as attributes [7]. Each OCT slice is flattened before to create a 3-level mutltiscale spatial pyramid. From each layer of this pyramid, edges are extracted and LBP descriptors are computed for the flattened slice and the edge map. All the obtained histograms are concatenated into a global descriptor whose dimensions are reduced using PCA. Finally, a SVM with a Radial Basis Function (RBF) kernel is used as classifier. A detection rate with an AUC of 0.93 is achieved, using a dataset of 326 OCT scans with various pathologies.

Albarrak et al. propose another classification framework to differentiate AMD and normal volumes [13]. Each OCT slice undergoes two pre-processing routines: (i) a joint denoising and cropping step using the split Bregman isotropic total variation algorithm and (ii) a flattening step by fitting a second-order polynomial using a least-square approach. Then, LBP-TOP and HOG combined with LBP-TOP features are extracted from individual sub-volumes from each original cropped volume. These features are concatenated into a single feature vector per OCT volume and its dimension is reduced using PCA. Finally, a Bayesian network classifier is used to classify the volumes. The classification performance of the framework in terms of SE and SP achieves $92.4 \%$ and $90.5 \%$, respectively, outperforming the method of Liu et al. [7], using a dataset composed of 140 OCT volumes.

Anantrasirichai et al. propose to detect glaucoma in OCT images based on a variety of texture descriptor [14]. The texture information is described through LBP, Gray-level cooccurrence matrix (GLCM), wavelet, granulometry, run length measures, and intensity level distributions in combination with retinal layer thickness estimation, without any pre-processing. Each feature vector is projected using PCA before to be classified using an SVM with both linear and RBF kernel. Testing with rather a small dataset of 24 OCT volumes, their 
proposed method achieves an Accuracy (ACC) of $85 \%$ while using layer thickness and textural informations.

\section{B. Semi-supervised methods}

Sankar et al. propose to use a semi-supervised strategy to classify DME vs. normal OCT volumes based on appearance modeling of normal OCT images using Gaussian Mixture Model (GMM) [15]. The main difference between this method and the supervised methodologies lies in the fact that only normal volumes are used to train the system.

For each OCT volume, the B-scans are denoised using NLM filtering, flattened, and resized to ensure homogeneous dimension across all volumes. Each B-scan is vectorized and projected into a lower-dimensional space with $p$ dimensions using PCA. Subsequently, normal B-scans are modelled using a GMM in which the number of mixture components $K$ is determined on a validation set. At the testing stage, a scan is classified as normal or DME depending of its Mahalanobis distance to the learnt model; if the distance is greater than the $97.5 \%$ quantile of the Chi-squared distribution with $p$ degree of freedom. Therefore, a volume is classified as abnormal if the number of abnormal slice is greater than a given threshold, previously determined during the validation procedure. A SE and SP of $93.8 \%$ and $80.0 \%$ are respectively achieved on a cohort of 32 patients.

\section{DATA}

Proper comparison of different methodologies require a common dataset to test these methodologies. The lack of public data for comparing methodologies is recurrent claim in the medical image community [17]. To amend this limitation, Duke Univeristy made two SD-OCT datasets available to the ophtalmic community [9, 10].

The rest of this section highlights the advantages and disadvantages of the datasets provided by Duke University, points out why this datasets cannot be used for our purposes and finally describes our data: the SERI dataset [18].

The former dataset from Duke University consists of 384 OCT annotated volumes classified either as AMD or normal cases. Despite the advantage of testing in large datasets, this dataset cannot be used to conduct our study since we are interested in DME and not AMD. This dataset has been used by Venhuizen et al. [6] to test their method since their main interest is AMD detection. The later dataset from Duke Univeristy consists of 45 pre-processed OCT volumes and labeled as AMD, DME, and normal. Despite this dataset is suitable to our goal of classifying DME vs normal volumes, the dataset has been dropped since there is no access to the original data. All volumes have been denoised, aligned and cropped. This dataset has been used to conduct the experimentation reported by Srinivasan et al. [10].

The dataset to conduct our study has been acquired by the SERI, using CIRRUS TM (Carl Zeiss Meditec, Inc., Dublin, CA) SD-OCT device. This dataset consists of 32 OCT volumes, subdivided into $16 \mathrm{DME}$ and 16 normal cases. Each volume contains 128 B-scans with a resolution of
$512 \mathrm{px} \times 1,024 \mathrm{px}$. All SD-OCT images have been read and assessed by trained graders and identified as normal or DME cases, based on evaluation of retinal thickening, hard exudates, intraretinal cystoid space formation and subretinal fluid (see Fig.2).

\section{EXPERIMENTAL SETUP}

The experimental set-up is summarized in Table I] where the most relevant works in Sect.[I] are formulated as the 5-steps standard classification procedure described in Fig. 1 .

\section{A. Implementation details}

The experiments, described in this work, are publicly available at [19] allowing for further comparisons and improvements. All the methods in Table I have been developed using protoclass [20], a rapid prototyping toolkit to perform image processing and Machine Learning (ML) tasks. Furthermore, each method has been implemented as a plug-in to [19], so that all methods can be evaluated in a common framework 1

Note that Liu et al. train the algorithm at the B-scan level, and SERI dataset provides Ground Truth (GT) at volume level only. Thus, two strategies have been explored to solve this issue: (i) similarly to Srinivasan et al., at training stage, all Bscans are considered as abnormal for a DME volume and at testing stage, a majority vote rule is applied to whether label a volume as abnormal or not; (ii) similarly to Venhuizen et al., an approach using BoW is used. From the methods reviewed in Sect.II] we decline to implement Albarrak et al. and Anantrasirichai et al. . The former do not provide sufficient implementation details to replicate their results [13]; while, the latter use a descriptor based on the layer thickness which require a layer segmentation stage using a generic segmentation algorithm and further user validation [14].

\section{B. Evaluation}

All the experiments are evaluated in terms of SE and SP (see Fig. 3) using the Leave-Two-Patient Out Cross-Validation (LTPO-CV) strategy ${ }^{2} \mathrm{SE}$ evaluates the performance of the classifier with respect to the positive class, while the SP does the same with respect to negative class. LTPO-CV keeps two volumes (one normal and one DME) for testing while the remaining volumes are used as training. The advantage of using LTPO-CV over Leave-One-Patient Out Cross-Validation (LOPO-CV) is that LTPO-CV keeps the training data balanced. The main drawback of using LTPO-CV (or LOPO$\mathrm{CV}$ ) is that despite reporting robust performance estimators, variance of this descriptors cannot be computed. Despite this limitation, LTPO-CV strategy has been adopted here, since regular Computer Vision (CV) cannot be applied due to the reduced size of the dataset.

\footnotetext{
${ }^{1}$ See table I for standalone repositories of each method. All repositories provide tests to ensure that our implementation comply with the original work. ${ }^{2}$ the same evaluation strategy was applied [12]
} 


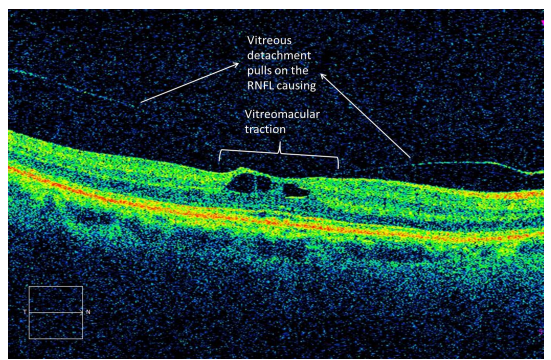

(a) Vitreomacular traction.

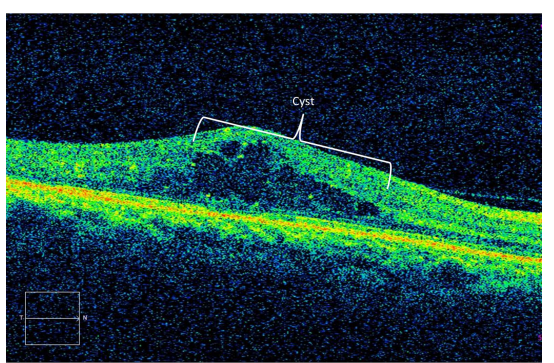

(d) Cyst spaces and hard exudates, causing central retinal thickening.

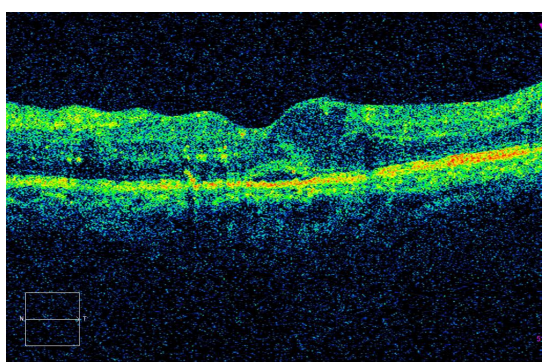

(g) Cyst spaces, causing retinal thickening.

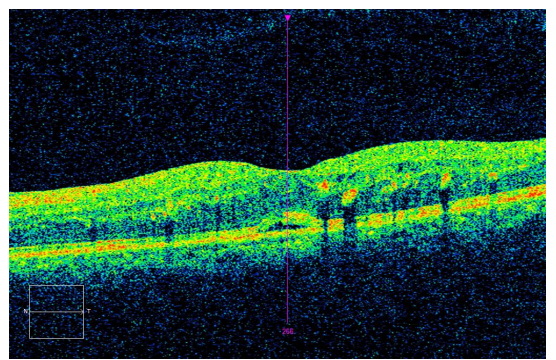

(b) Rethinal thickening.

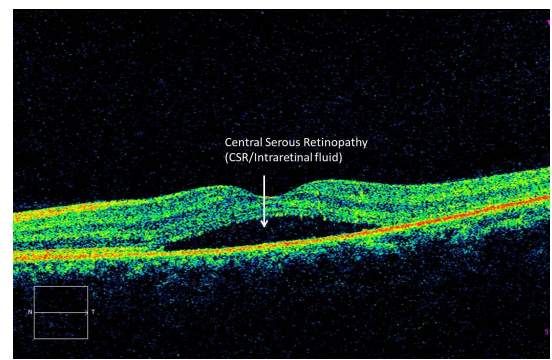

(e) CSR (subretinal fluid), causing central and parafoveal thickening.

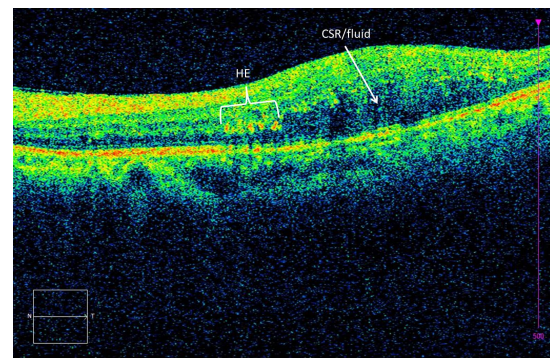

(h) CSR and hard exudates, causing retinal thickening.

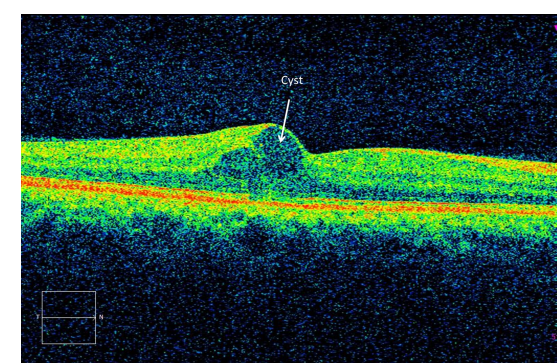

(c) Cyst spaces, causing central and parafoveal retina thickening.

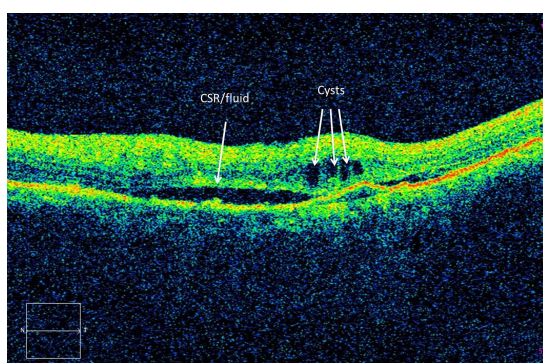

(f) CSR, hard exudates and cyst spaces.

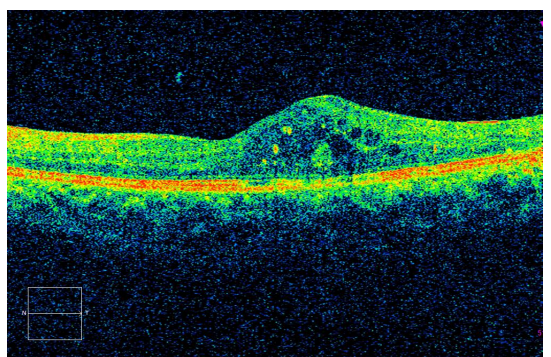

(i) Cyst spaces causing parafoveal thickening.

Fig. 2. Examples of DME cases in Singapore Eye Research Institute (SERI) dataset.

\section{REsUlts AND DisCUSSION}

The entire set of experiments with their associated results can be found in [19], while Table I] shows the configuration leading to the best results of each method. The results are reported in terms of SE and SP (see Sect.IV-B).

Lemaitre et al. achieve the best results when using LBPTOP features, a global mapping, and histogram representation [12]. Alsaih et al. perform better when using HOG features with PCA representation [16]. Our interpretation of Liu et al., as proposed in Sect.IV achieves the best results when using majority voting instead of BoW models. Refer to Table 1 for configuration details of the remaining methods.

Results in [19] indicate two main findings with major impact: (i) features describing the entire volume rather than each B-scan are more discriminative; and (ii) a pre-processing stage with denoising is fundamental.

Other observations include the facts that (i) to represent Bscans, local mapping in conjunction with dimension reduction, either using PCA or BoW, improve the results. However, the combination of both decreases the performance in comparison to non reduced histogram representation; (ii) building BoW models from the concatenation of all features for each B-scan, might lead to the curse of dimensionality since 128 samples per volume is not enough to describe a space with a number of dimensions of the order of thousands; which could explain the over-fitting using RBF-SVM as in [21].

\section{CONCLUSION AND FURTHER WORK}

The work here presented states the relevance of developing methodologies to automatically differentiate DME vs. normal SD-OCT scans. This article offers an overview of the state-ofthe-art of DME detection and provides a public benchmarking to facilitate further studies. In this regard, there are two crucial aspects to improve the work here presented: (i) enlarge the dataset. (ii) reach out to other authors in order to enlarge this benchmark with additional methods and improve the existing approaches. 
TABLE I

CORRESPONDENCE BETWEEN THE MOST RELEVANT METHODOLOGIES REVIEWED IN SECT. [IAND THE PROPOSED EXPERIMENTAL FRAMEWORK.

\begin{tabular}{|c|c|c|c|c|c|}
\hline Ref & Pre-processing & Features & Mapping & Representation & Classification \\
\hline Venhuizen et al. 6 22] & - & Texton & Local & $\begin{array}{l}\text { PCA } \\
\text { BoW }\end{array}$ & $\mathrm{RF}$ \\
\hline Srinivasan et al. 10 23 & $\begin{array}{c}\text { Denoising (BM3D) } \\
\text { Flattening } \\
\text { Cropping }\end{array}$ & HOG & Global & - & $\begin{array}{l}\text { Linear- } \\
\text { SVM }\end{array}$ \\
\hline Lemaître et al. [12 24] & Denoising (NLM) & $\begin{array}{c}\text { LBP } \\
\text { LBP-TOP }\end{array}$ & $\begin{array}{l}\text { Local } \\
\text { Global }\end{array}$ & $\begin{array}{c}\text { PCA } \\
\text { BoW } \\
\text { Histogram }\end{array}$ & $\mathrm{RF}$ \\
\hline Alsaih et al. [11, 16] & $\begin{array}{c}\text { Denoising (BM3D) } \\
\text { Flattening } \\
\text { Cropping }\end{array}$ & $\begin{array}{l}\text { LBP } \\
\text { HOG }\end{array}$ & Local & $\begin{array}{c}\text { PCA } \\
\text { Histogram }\end{array}$ & $\begin{array}{l}\text { Linear- } \\
\text { SVM }\end{array}$ \\
\hline Liu et al. 721 & $\begin{array}{l}\text { Flatten } \\
\text { Aligned }\end{array}$ & $\begin{array}{l}\text { Edge } \\
\text { LBP }\end{array}$ & Local & $\begin{array}{l}\text { PCA } \\
\text { BoW }\end{array}$ & $\begin{array}{l}\text { RBF- } \\
\text { SVM }\end{array}$ \\
\hline $\begin{array}{l}-------- \\
\text { Sankar et al. [15 25 }\end{array}$ & $\begin{array}{l}\text { Denoising (NLM) } \\
\text { Flattening } \\
\text { Cropping }\end{array}$ & $\begin{array}{l}-\overline{-}-- \\
\text { Pixel } \\
\text { intensities }\end{array}$ & Global & $\begin{array}{c}----- \\
\text { PCA }\end{array}$ & $\begin{array}{c}\text { Mahalanobis } \\
\text {-distance } \\
\text { to GMM }\end{array}$ \\
\hline
\end{tabular}

TABLE II

SUMMARY OF THE CLASSIFICATION PERFORMANCE IN TERMS OF SE AND SP IN (\%).

\begin{tabular}{ccccccc}
\hline & Lemaitre et al. [12] & Sankar et al. [15] & Alsaih et al. [16] & Srinivasan et al. [10] & Liu et al. [7] & Venhuizen et al. [6] \\
\hline SE & 87.5 & 81.3 & 75.0 & 68.8 & 68.8 & 61.5 \\
SP & 75.0 & 62.5 & 87.5 & 93.8 & 93.8 & 58.8 \\
\hline
\end{tabular}

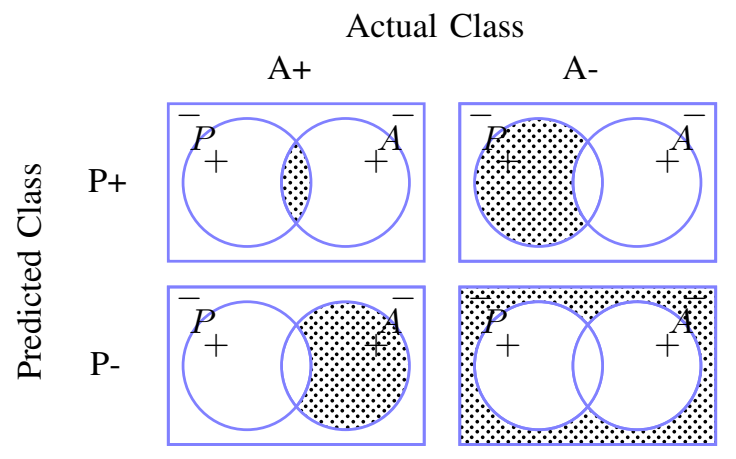

(a) Confusion matrix with truly and falsely positive samples detected (TP, FP) in the first row, from left to right and the falsely and truly negative samples detected $(\mathrm{FN}, \mathrm{TN})$ in the second row, from left to right.

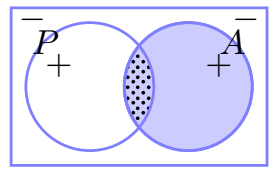

$$
S E=\frac{T P}{T P+F N}
$$

(b) SE and SP evaluation, corresponding to the ratio of the doted area over the blue area.

Fig. 3. Evaluation metrics: (a) confusion matrix, (b) SE - SP

\section{REFERENCES}

[1] S. Wild, G. Roglic, A. Green, R. Sicree, and H. King, "Global prevalence of diabetes estimates for the year 2000 and projections for 2030," Diabetes care, vol. 27, no. 5, pp. 1047-1053, 2004.

[2] Early Treatment Diabetic Retinopathy Study Group, "Photocoagulation for diabetic macular edema: early treatment diabetic retinopathy study report no 1," JAMA Ophthalmology, vol. 103, no. 12, pp. 1796-1806, 1985.

[3] Y. T. Wang, M. Tadarati, Y. Wolfson, S. B. Bressler, and N. M. Bressler, "Comparison of Prevalence of Diabetic Macular Edema Based on Monocular Fundus Photography vs Optical Coherence Tomography," JAMA Ophthalmology, pp. 1-7, Dec 2015.

[4] S. J. Chiu, X. T. Li, P. Nicholas, C. A. Toth, J. A. Izatt, and S. Farsiu, "Automatic segmentation of seven retinal layers in sd-oct images congruent with expert manual segmentation," Optic Express, vol. 18, no. 18, pp. 19413-19428, 2010

[5] R. Kafieh, H. Rabbani, M. D. Abramoff, and M. Sonka, "Intra-retinal layer segmentation of $3 \mathrm{~d}$ optical coherence tomography using coarse grained diffusion map," Medical Image Analysis, vol. 17, pp. 907-928, 2013.

[6] F. G. Venhuizen, B. van Ginneken, B. Bloemen, M. J. P. P. van Grisven, R. Philipsen, C. Hoyng, T. Theelen, and C. I. Sanchez, "Automated agerelated macular degeneration classification in OCT using unsupervised feature learning," in SPIE Medical Imaging, vol. 9414, 2015, p. 941411.

[7] Y.-Y. Liu, M. Chen, H. Ishikawa, G. Wollstein, J. S. Schuman, and R. J. M., "Automated macular pathology diagnosis in retinal oct images using multi-scale spatial pyramid and local binary patterns in texture and shape encoding," Medical Image Analysis, vol. 15, pp. 748-759, 2011.

[8] J. S. Schuman, C. A. Puliafito, J. G. Fujimoto, and J. S. Duker, Optical coherence tomography of ocular diseases. SLACK incorporated, 2004.

[9] S. Farsiu, S. J. Chiu, R. V. O'Connell, F. A. Folgar, E. Yuan, J. A. Izatt, C. A. Toth, A.-R. E. D. S. . A. S. D. O. C. T. S. Group et al., "Quantitative classification of eyes with and without intermediate 
age-related macular degeneration using optical coherence tomography,' Ophthalmology, vol. 121, no. 1, pp. 162-172, 2014

[10] P. P. Srinivasan, L. A. Kim, P. S. Mettu, S. W. Cousins, G. M. Comer J. A. Izatt, and S. Farsiu, "Fully automated detection of diabetic macular edema and dry age-related macular degeneration from optical coherence tomography images," Biomedical Optical Express, vol. 5, no. 10, pp. 3568-3577, 2014

[11] K. Alsaih, G. Lemaître, J. Massich, M. Rastgoo, D. Sidibé, T. Y. Wong, E. Lamoureux, D. Milea, C. Y. Cheung, and F. Mériaudeau, "Classification of sd-oct volumes with multi pyramids, lbp and hog descriptors: application to dme detections," in 38th IEEE Engineering in Medicine and Biology Society (EMBC). Orlando, USA: IEEE, 2016.

[12] G. Lemaitre, M. Rastgoo, J. Massich, S. Sankar, F. Meriaudeau, and D. Sidibe, "Classification of SD-OCT volumes with LBP: Application to dme detection," in Medical Image Computing and ComputerAssisted Intervention(MICCAI), Ophthalmic Medical Image Analysis Workshop (OMIA), 2015

[13] A. Albarrak, F. Coenen, and Y. Zheng, "Age-related macular degeneration identification in volumetric optical coherence tomography using decomposition and local feature extraction," in Proceedings of 2013 International Conference on Medical Image, Understanding and Analysis, 2013, pp. 59-64

[14] N. Anantrasirichai, A. Achim, J. E. Morgan, I. Erchova, and L. Nicholson, "Svm-based texture classification in optical coherence tomography," in IEEE 10th International Symposium on Biomedical Imaging (ISBI). IEEE, 2013, pp. 1332-1335.

[15] S. Sankar, D. Sidibé, Y. Cheung, T. Wong, E. Lamoureux, D. Milea, and F. Meriaudeau, "Classification of sd-oct volumes for dme detection: an anomaly detection approach," in SPIE Medical Imaging. International Society for Optics and Photonics, 2016, pp. 97 852O-97852O.

[16] K. Alsaih, G. Lemaitre, J. Massich, M. Rastgoo, D. Sidibe, and F. Meriaudeau, "Classification of SD-OCT volumes with multi pyramids, LBP and HOG descriptors: application to DME detections,' Aug. 2016. [Online]. Available: http://dx.doi.org/10.5281/zenodo.50145
[17] M. L. Giger, H.-P. Chan, and J. Boone, "Anniversary paper: History and status of CAD and quantitative image analysis: the role of medical physics and AAPM," Medical physics, vol. 35, no. 12, p. 5799, 2008

[18] G. Lemaitre, J. Massich, M. Rastgoo, and F. Meriaudeau, "Seri dataset," Sep. 2016. [Online]. Available: http://i2cvb.github.io/\#retino

[19] G. Lemaitre, J. Massich, and M. Rastgoo, "I2Cvb Benchmark: automatic classification of DME vs normal SD-OCT volumes," Apr 2016. [Online]. Available: http://dx.doi.org/10.5281/zenodo.61976

[20] G. Lemaitre, "Protoclass, a rapid prototyping tool for image processing and machine learning," Apr. 2016. [Online]. Available: http://glemaitre.github.io/protoclass/

[21] G. Lemaitre, J. Massich, and M. Rastgoo, "liu-2011-oct: ICPR2016," Apr. 2016. [Online]. Available: http://dx.doi.org/10.5281/zenodo.50140

[22] — "venhuizen-2015-feb: Icpr2016," Feb. 2016. [Online]. Available: http://dx.doi.org/10.5281/zenodo.61981

[23] G. Lemaitre, "srinivasan-2014-oct: Icpr 2016," Apr. 2016. [Online]. Available: http://dx.doi.org/10.5281/zenodo.50136

[24] G. Lemaitre, J. Massich, and M. Rastgoo, "retinopathy: Jo-omia-2015," Nov. 2015. [Online]. Available: http://dx.doi.org/10.5281/zenodo.34277

[25] G. Lemaitre, J. Massich, M Rastgoo, S. Sankar, D. Sidibe, and F. Meriaudeau, "sankar-2016-feb: Icpr2016," Feb. 2016. [Online]. Available: http://dx.doi.org/10.5281/zenodo.50137 